\title{
Changes in matrix protein biochemistry and the expression of mRNA encoding matrix proteins and metalloproteinases in posterior tibialis tendinopathy
}

\author{
Anthony N Corps, ${ }^{1}$ Andrew H N Robinson, ${ }^{2}$ Rebecca L Harrall, ${ }^{1}$ Nicholas C Avery, ${ }^{3}$ \\ Valerie A Curry, ${ }^{1}$ Brian L Hazleman, ${ }^{1}$ Graham P Riley ${ }^{4}$
}

- Additional data are published online only. To view the files please visit the journal online (http://ard.bmj.com/ content/71/5.toc)

${ }^{1}$ Rheumatology Research Unit, Addenbrooke's Hospital, Cambridge, UK

2Department of Trauma and Orthopedics, Addenbrooke's Hospital, Cambridge, UK

${ }^{3}$ Matrix Biology Research Group, School of Veterinary Sciences,

University of Bristol, UK

${ }^{4}$ School of Biological Sciences, University of East Anglia, Norwich, UK

\section{Correspondence to}

Graham Peter Riley, School of Biological Sciences, University of East Anglia, Earlham Road, Norwich NR4 7TJ, UK; graham.riley@uea.ac.uk

Received 6 July 2011 Accepted 29 November 2011 Published Online First 12 January 2012

\section{ABSTRACT \\ Objectives Adult-acquired flat foot secondary to a dysfunctional posterior tibialis tendon (PTT) is often treated by surgical transfer of the flexor digitorum longus tendon (FDLT). In this study, the authors compared normal PTT, stage II dysfunctional PTT and replacement FDLT, aiming to define changes in collagen modification, glycosaminoglycan (GAG) and the expression of matrix and metalloproteinase mRNA.}

Methods Normal PTTs were obtained from patients with no history of tendon problems. Samples of dysfunctional PTT and replacement FDLT tissue were obtained from patients undergoing surgical reconstruction. Tissue samples were analysed for total collagen and GAG, pentosidine and collagen cross-links. Total RNA was assayed for mRNA encoding matrix proteins and metalloproteinases, using real-time reverse transcription PCR. Differences between clinical groups were assessed using non-parametric statistics.

Results Dysfunctional PTT contained higher levels of GAG and lower levels of pentosidine than normal PTT or FDLT. In contrast, collagen in FDLT contained fewer ketoimine and more aldimine cross-links than either normal or dysfunctional PTT. mRNA encoding types I and III collagens, aggrecan, biglycan, matrix metalloproteinase (MMP)-2, -13 and -23 , and a disintegrin and metalloproteinase (ADAM)-12L each showed increased levels in dysfunctional PTT compared with either normal PIT or (except MMP-13) FDLT. In contrast, MMP-3 and ADAM with thrombospondin domain (ADAMTS)-5 mRNA were lower in both dysfunctional PTT and FDLT than in normal PTT, while ADAMTS-1 mRNA was lower in dysfunctional PTT than in FDLT.

Conclusions Stage II dysfunctional PTT shows biochemical and molecular changes consistent with a chronic remodelling of the extracellular matrix, rather than rupture, while the replacement FDLT resembles normal PTT in many, but not all, parameters.

\section{INTRODUCTION}

Dysfunction of the human posterior tibialis tendon (PTT) is a common cause of adult-acquired flat foot. PTT disease particularly affects middle-aged women and causes a significant proportion of the tendon pathology seen in the clinic. ${ }^{1-5}$ In stage II dysfunction, in which the foot retains flexibility and the defect is correctible, the PTT is elongated and shows marked degeneration, but is not generally ruptured. ${ }^{2-5}$ The preferred surgical treatment involves tendon transfer to restore functional support of the foot arch, normally using the neighbouring flexor digitorum longus tendon (FDLT), with a calcaneal osteotomy to enhance biomechanical performance. $^{25-7}$

The extracellular matrix of tendons varies between different tendons and regionally within tendons, and there are changes in tendinopathy or ruptured tendons compared with normal tendons. ${ }^{8} 9$ There is a linear, age-dependent accumulation of collagen modifications in human biceps brachii and patellar tendons that indicates a low rate of turnover of the matrix. ${ }^{10-12}$ The absence of an equivalent accumulation of modifications in supraspinatus tendons is consistent with a higher level of collagen replacement in these injury-prone tendons. ${ }^{10}$ A difference in collagen turnover rates has also been described recently in equine common digital extensor and superficial digital flexor tendons. ${ }^{13}$ In normal tendons, versican is the predominant large aggregating proteoglycan in the tensile mid-tendon, but aggrecan, which is characteristic of cartilage, is present in fibrocartilaginous regions where the tendons attach to bone or wrap around pivots. ${ }^{14} 15$ In chronic Achilles tendinopathy, increased glycosaminoglycan (GAG) levels in the morphologically abnormal mid-tendon indicate increased proteoglycan, ${ }^{16}$ and high levels of mRNA encoding aggrecan and biglycan are expressed. ${ }^{17}$ The observed differences in matrix protein composition may also involve changed expression and/ or activity of many enzymes of the metalloproteinase clan. ${ }^{18} 19$

In the normal PTT, as in other tendons, fibrocartilaginous regions enriched in aggrecan and type II collagen are found at the entheses and on the inner zone of the tendon where it wraps around the medial malleolus. ${ }^{20} 21$ In stage II PTT dysfunction, hypercellularity, increased vascularisation, increased mucin levels and a disrupted collagen matrix containing a higher proportion of types III and V collagens have been reported. ${ }^{22-24}$ Otherwise, however, little is known of the detailed biochemical and molecular changes underlying PTT dysfunction. In this study, we aimed to determine how biochemical markers of matrix composition, and the expression of mRNA encoding a range of matrix components and metalloproteinases, shown previously to change significantly in other tendinopathies, ${ }^{17} 19$ varied between normal and stage II dysfunctional PTT. Given that the PTT 
Table 1 Normal PTT, stage II PTT and FDLT samples

\begin{tabular}{|c|c|c|c|c|c|c|}
\hline & \multicolumn{3}{|c|}{ Women } & \multicolumn{3}{|c|}{ Men } \\
\hline & Normal PTT & Stage II PTT & FDLT & Normal PTT & Stage II PTT & FDLT \\
\hline Number of samples & 6 & $25^{*}$ & $25^{*}$ & 7 & 7 & 6 \\
\hline Age (years), median (range) & $53.5(29-87)$ & $60(30-77)$ & $60(30-77)$ & $54(35-63)$ & $55(19-72)$ & $56.5(54-72)$ \\
\hline \multicolumn{7}{|l|}{ Histology } \\
\hline Normal & $5 \dagger$ & 1 & $19 \dagger$ & 5 & 1 & 5 \\
\hline Abnormal & 0 & 24 & 5 & 2 & 6 & 1 \\
\hline \multicolumn{7}{|l|}{ Abnormal features } \\
\hline Fibre disorganisation & 0 & 24 & 0 & 0 & 6 & 0 \\
\hline Increased cellularity & 0 & 21 & 4 & 1 & 6 & 0 \\
\hline Cell rounding & 0 & 24 & 3 & 2 & 6 & 1 \\
\hline Increased GAG & 0 & 24 & 0 & 0 & 6 & 0 \\
\hline Blood vessel infiltration & 0 & 24 & 5 & 1 & 6 & 0 \\
\hline
\end{tabular}

Tendon samples were collected from patients as described in the text. Dissected pieces were fixed and examined histologically using standard techniques (see online supplementary text).

*The 25 samples of stage II PTT and FDLT are paired samples from the same individual female patients.

tIndicates that one each of these sample sets was not available for histology. Five abnormal features were scored: collagen

fibre disorganisation, increased cellularity, cell rounding, increased GAG content and blood vessel infiltration (indicating

neovascularisation or angiogenesis). The number of samples presenting normal and abnormal appearance is shown, together

with the numbers of samples showing each specific abnormality.

FDLT, flexor digitorum longus tendon; GAG, glycosaminoglycan; PTT, posterior tibialis tendon.

Table 2 Collagen content, GAG content and collagen modifications in normal PTT, stage II PTT and FDLT from female patients

\begin{tabular}{|c|c|c|c|c|c|c|}
\hline & \multicolumn{3}{|c|}{ Median (range) } & \multicolumn{3}{|c|}{ p Value } \\
\hline & Normal PTT & Stage II PTT & FDLT & N PTT v StIl PTT & N PTT v FDLT & StII PTT v FDLT \\
\hline \multicolumn{7}{|c|}{ Collagen and GAG ( $\mu \mathrm{g} / \mathrm{mg}$ dry weight) } \\
\hline \multicolumn{7}{|c|}{ Collagen modifications (mol/mol collagen) } \\
\hline Pentosidine & $4.2 \times 10^{-3}(2.1-7.4)$ & $1.6 \times 10^{-3}(0.3-4.2)$ & $5.8 \times 10^{-3}(3.3-10.8)$ & $\mathrm{p}<0.05$ & nsd & $p<0.001$ \\
\hline OHPyr & $0.48(0.26-0.59)$ & $0.38(0.27-0.50)$ & $0.13(0.05-0.24)$ & nsd & $p<0.001$ & $p<0.001$ \\
\hline HLNL & $0.10(0.06-0.17)$ & $0.11(0.05-0.16)$ & $0.22(0.11-0.33)$ & nsd & $\mathrm{p}<0.001$ & $\mathrm{p}<0.001$ \\
\hline
\end{tabular}

FDLT, flexor digitorum longus tendon; GAG, glycosaminoglycan; N, normal; nsd, no significant difference; PTT, posterior tibialis tendon; Stll, stage II. OHPyr, hydroxylysyl pyridinoline, HLKNL, hydroxylysino-5-ketonorleucine, HHL, histidinohydroxylysinonorleucine, HLNL, hydroxylysinonorleucine

and FDLT are functionally different tendons, and may therefore have different composition (see Discussion), we also assayed the same parameters in the FDLT used for reconstruction.

\section{METHODS}

\section{Tendon specimens}

Consistent with PTT dysfunction occurring more widely in women, the majority of patients presenting in this study were female (table 1). Samples of PTT and FDLT tissue were obtained from patients clinically staged as having stage II PTT dysfunction and being surgically treated with FDLT transfer. Specimens of normal PTT were obtained from patients with no history of tendon problems, either undergoing lower limb amputation for bone tumours (Royal National Orthopaedic Hospital, Stanmore) or with foot drop (usually resulting from a slipped intervertebral disc) being corrected by the transfer of normal PTT (Addenbrooke's Hospital, Cambridge). Procedures were approved by the relevant local research ethics committees, and written consent was obtained from informed patients.

Specimens of normal PTT and FDLT generally showed normal tendon organisation, whereas specimens from patients with stage II PTT dysfunction showed features typical of tendinopathy (table 1). ${ }^{1622} 2425$ Although morphologically normal, we could not exclude the possibility that the phenotype of the normal PTT may have been affected by reduced weight-bearing and mobility, and/or chemotherapy.

\section{Histological and biochemical characterisation, isolation of total RNA, and analysis by relative quantitative PCR}

See online supplementary text for a description of these methods.

\section{Statistical analysis}

Differences between clinical groups are described as n-fold differences between the medians and were analysed using either the Mann-Whitney $U$ test or, for the paired samples of dysfunctional PTT and FDLT, the Wilcoxon signed rank test.

\section{RESULTS}

Biochemical analysis of PTT and FDLT from female patients Tendon collagen and proteoglycan content

The median collagen content of normal PTT, dysfunctional PTT and FDLT samples from female patients showed no significant differences (table 2). In contrast, significant differences in proteoglycan content were observed: dysfunctional PTT contained twofold and sixfold higher levels of GAG than normal PTT and FDLT, respectively. The threefold higher level of GAG in normal PTT compared with FDLT was also significant (table 2).

Pentosidine modification and cross-linking of collagen

Normal PTT showed a linear increase in pentosidine (non-enzymatic glycation of collagen) with increasing age of the donor, and FDLT generally showed a similar pentosidine content to normal 
(A) Pentosidine

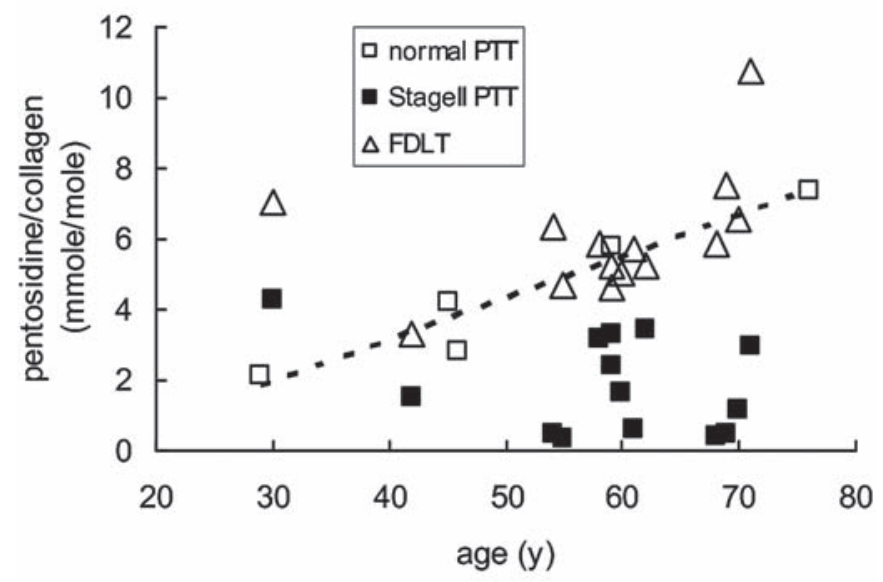

(B) OHPyr

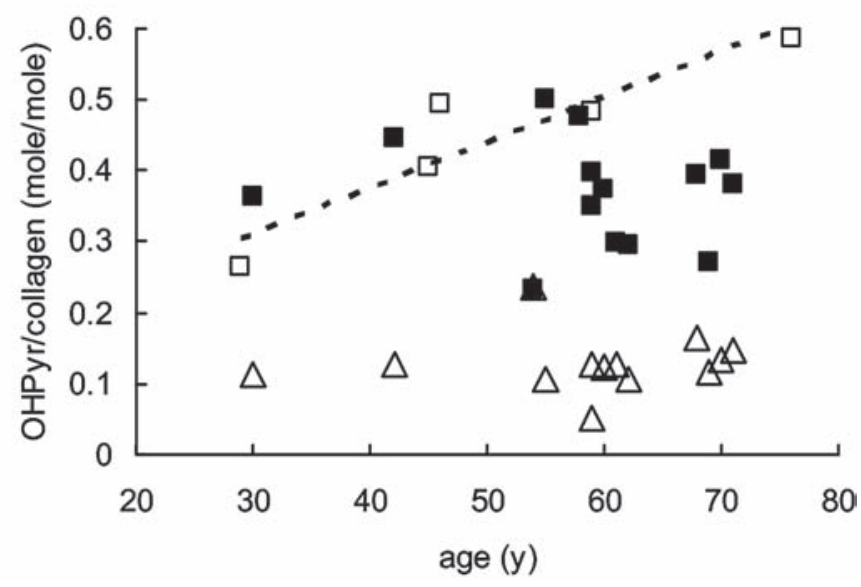

Figure 1 Accumulation of pentosidine and hydroxylysyl pyridinoline cross-links in collagen in female posterior tibialis tendon (PTT) and flexor digitorum longus tendon (FDLT) as a function of age. (A) Pentosidine and (B) OHPyr cross-links were determined by HPLC as outlined in the text, and plotted against the age of the donor. Each point represents an individual sample. The broken line shows linear regression analysis based on the normal PTT samples: $(A) R^{2}=0.92$, $p=0.01 ;(B) R^{2}=0.85, p=0.02$.
PTT of equivalent age (figure 1A). In contrast, dysfunctional PTT collagen contained significantly lower levels of pentosidine than that predicted by age from normal PTT or paired FDLT samples (figure 1A, table 2).

When the pattern of collagen cross-linking was considered, FDLT differed markedly from both normal and dysfunctional PTT (table 2). Ketoimine cross-link levels were lower in FDLT than in either normal or dysfunctional PTT; this was due to differences in hydroxylysyl pyridinoline (OHPyr) levels, not in hydroxylysino-5-ketonorleucine (HLKNL) (table 2), and applied across the age range (figure $1 \mathrm{~B}$ ). In contrast, aldimine cross-link levels were higher in FDLT than in either the normal or dysfunctional PTT, and this was due to differences in both histidinohydroxylysinonorleucine (HHL) and hydroxylysinonorleucine (HLNL) levels (table 2). Similarly to the results for the pentosidine modification, OHPyr levels in normal PTT showed some increase with age, and samples of dysfunctional PTT contained lower levels of OHPyr than predicted by age from normal PTT (figure 1B).

\section{mRNA expression in PTT and FDLT from female patients}

Of the mRNA assayed, only matrix metalloproteinase (MMP)-2 mRNA showed a significant change with increasing age, a small increase in normal PTT (data not shown); however, this could not account for the difference observed between normal and dysfunctional PTT (see below).

\section{mRNA encoding collagens and proteoglycans}

mRNA of each of the major extracellular matrix proteins was readily detected in all samples of PTT and FDLT (table 3, figures 2 and 3$)$. mRNA of the small leucine-rich proteoglycan, decorin, was expressed at the highest levels $\left(\sim 10^{-3}\right.$ relative to $18 \mathrm{~S}$ rRNA), and showed no significant differences between the three clinical groups (figure $2 \mathrm{~A}$ ). mRNA of the large aggregating proteoglycan, versican, also showed no difference between normal and dysfunctional PTT, but showed $60 \%$ higher expression in dysfunctional PTT than in FDLT (figure 2C). Types I and III collagens ( $\alpha 1$ chains), aggrecan and biglycan each showed between threefold and 10-fold higher mRNA expression in dysfunctional PTT than in either normal PTT or FDLT, which were not significantly different from each other (table 3 ; figures $2 \mathrm{~B}$ and $3 \mathrm{~A})$.

Table 3 mRNA expression in normal PTT, stage II PTT and FDLT from female patients

\begin{tabular}{|c|c|c|c|c|c|c|}
\hline \multirow[b]{2}{*}{ Gene } & \multicolumn{3}{|c|}{ mRNA/18S } & \multicolumn{3}{|c|}{ p Value } \\
\hline & Normal PTT & Stage II PTT & FDLT & N PTT v Stll PTT & N PTT v FDLT & Stll PTT v FDLT \\
\hline Type I collagen & $7.6 \times 10^{-05}$ & $72.0 \times 10^{-05}$ & $20.6 \times 10^{-05}$ & $\mathrm{p}<0.001$ & nsd & $\mathrm{p}<0.001$ \\
\hline Type III collagen & $3.8 \times 10^{-05}$ & $24.4 \times 10^{-05}$ & $9.0 \times 10^{-05}$ & $\mathrm{p}<0.01$ & nsd & $\mathrm{p}<0.05$ \\
\hline Aggrecan & $5.3 \times 10^{-06}$ & $28.3 \times 10^{-06}$ & $4.8 \times 10^{-06}$ & $p=0.01$ & nsd & $p<0.001$ \\
\hline Versican & $1.9 \times 10^{-05}$ & $1.6 \times 10^{-05}$ & $0.9 \times 10^{-05}$ & nsd & nsd & $p<0.01$ \\
\hline Biglycan & $4.7 \times 10^{-05}$ & $20.8 \times 10^{-05}$ & $4.7 \times 10^{-05}$ & $p<0.05$ & nsd & $\mathrm{p}<0.001$ \\
\hline Decorin & $1.4 \times 10^{-03}$ & $0.8 \times 10^{-03}$ & $0.6 \times 10^{-03}$ & nsd & nsd & nsd \\
\hline MMP-1 & $5.8 \times 10^{-09}$ & $\left(<2 \times 10^{-09}\right)^{*}$ & $\left(<2 \times 10^{-09}\right)^{*}$ & nsd & nsd & nsd \\
\hline MMP-2 & $1.3 \times 10^{-05}$ & $5.5 \times 10^{-05}$ & $1.5 \times 10^{-05}$ & $p<0.01$ & nsd & $p<0.01$ \\
\hline MMP-3 & $1.7 \times 10^{-05}$ & $0.1 \times 10^{-05}$ & $0.1 \times 10^{-05}$ & $p=0.01$ & $\mathrm{p}<0.01$ & nsd \\
\hline MMP-13 & $\left(<2 \times 10^{-09}\right)^{*}$ & $41.6 \times 10^{-09}$ & $8.8 \times 10^{-09}$ & $p<0.05$ & nsd & nsd \\
\hline MMP-23 & $2.0 \times 10^{-08}$ & $33.4 \times 10^{-08}$ & $15.0 \times 10^{-08}$ & $p<0.05$ & nsd & $p<0.01$ \\
\hline ADAM-12L & $4.6 \times 10^{-08}$ & $73.5 \times 10^{-08}$ & $40.7 \times 10^{-08}$ & $p=0.01$ & nsd & $p<0.01$ \\
\hline ADAMTS-1 & $1.8 \times 10^{-06}$ & $0.9 \times 10^{-06}$ & $2.6 \times 10^{-06}$ & nsd & nsd & $p<0.001$ \\
\hline ADAMTS-4 & $2.8 \times 10^{-07}$ & $4.8 \times 10^{-07}$ & $4.1 \times 10^{-07}$ & nsd & nsd & $p<0.01$ \\
\hline ADAMTS-5 & $2.3 \times 10^{-06}$ & $0.9 \times 10^{-06}$ & $0.9 \times 10^{-06}$ & $p=0.001$ & $\mathrm{p}<0.01$ & nsd \\
\hline
\end{tabular}

Tendon RNA samples were assayed for specific mRNA, relative to 18S rRNA as described in the text, and the median value for each group is shown.

*Indicates that this mRNA was below the detection limit in the majority of the samples in this group.

ADAM, a disintegrin and metalloproteinase; ADAMTS, ADAM with thrombospondin domain; FDLT, flexor digitorum longus tendon; MMP, matrix metalloproteinase; N, normal; nsd, no significant difference; PTT, posterior tibialis tendon; Stll, stage II. 
(A) decorin

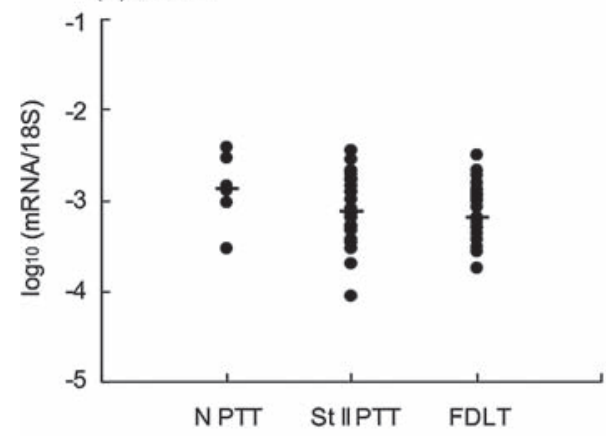

(C) versican

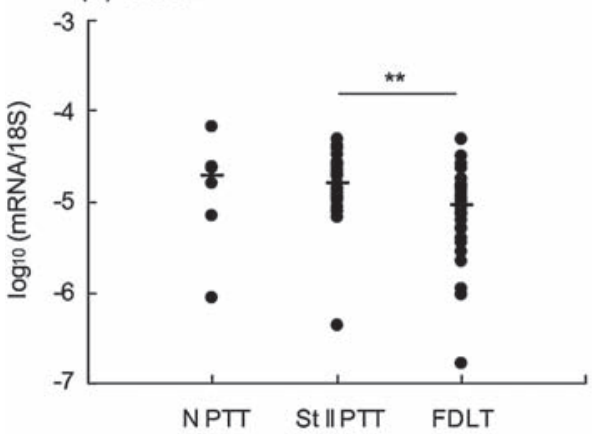

(E) ADAMTS-5

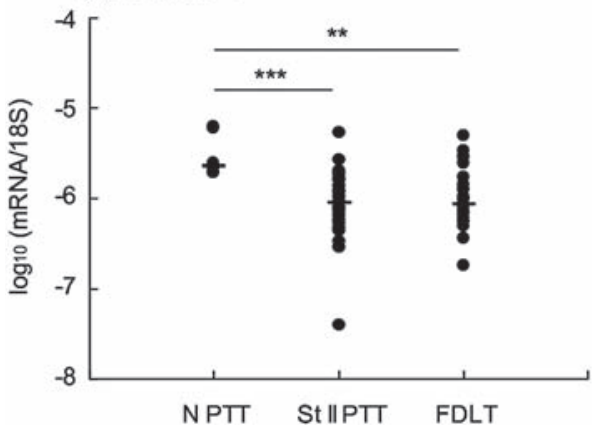

(B) aggrecan

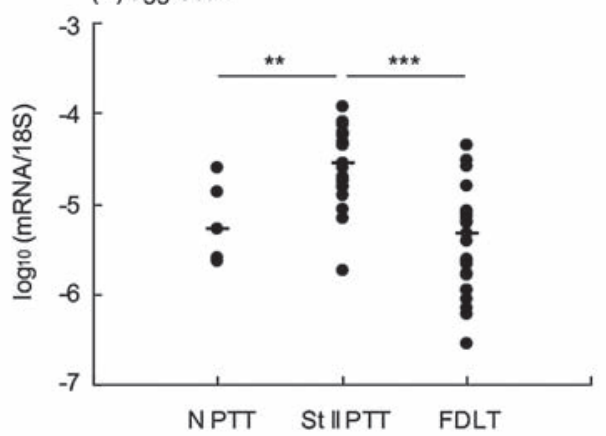

(D) MMP-13

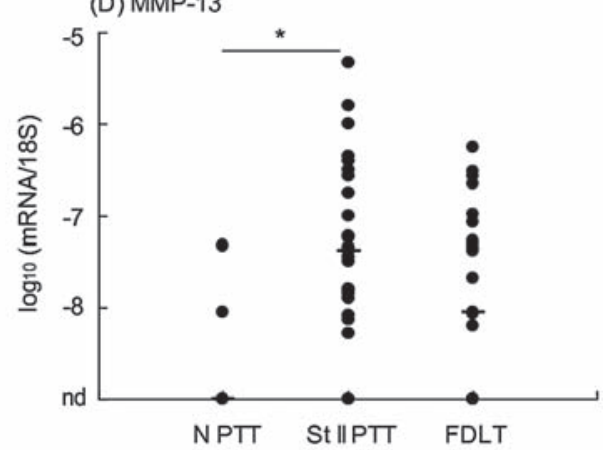

(F) ADAMTS-1

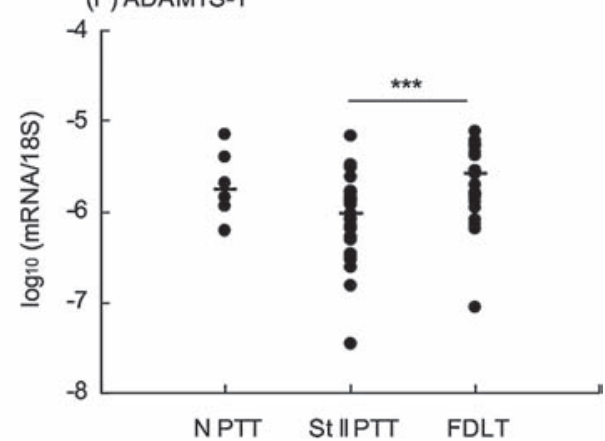

Figure 2 Expression of mRNA in female posterior tibialis tendon (PTT) and flexor digitorum longus tendon (FDLT). Tendon RNA samples were analysed for specific mRNA, expressed relative to 18S rRNA as outlined in the text. Each point represents an individual sample. nd (y-axis) indicates that the mRNA was not detectable (below $2 \times 10^{-9}$ relative to $18 \mathrm{~S}$ ) in one or more samples. The median values are shown (horizontal bar). ${ }^{* * *} p<0.001$, ${ }^{* *} \mathrm{p}<0.01,{ }^{*} \mathrm{p}<0.05$, for differences between the indicated clinical groups. ADAMTS, a disintegrin and metalloproteinase with thrombospondin domain; MMP, matrix metalloproteinase; N, normal; St II, stage II.

mRNA encoding metalloproteinases

In normal PTT, MMP-2 and MMP-3 were the most highly expressed of the metalloproteinase mRNAs, while mRNA levels of the collagenases, MMP-1 and MMP-13, were low or undetectable (table 3 ). The expression of mRNA of MMP-2, MMP-23 and a disintegrin and metalloproteinase (ADAM)-12L were each increased between fourfold and 16-fold in dysfunctional PTT compared with normal PTT, and there was detectable expression of MMP-13 in most dysfunctional PTT (table 3; figures 2D and $3 \mathrm{~B}$ ). mRNA levels of MMP-2, MMP-23 and ADAM-12L were also higher in dysfunctional PTT than in FDLT (table 3). In contrast with the other MMPs, expression of MMP-3 mRNA had decreased 15-fold in both dysfunctional PTT and FDLT compared with normal PTT, but there was no significant difference between dysfunctional PTT and FDLT (table 3; figure 3C).

mRNA expression of the three major ADAMTS (a disintegrin and metalloproteinase with thrombospondin domain) aggrecanases showed smaller changes than the other metalloproteinases, and each ADAMTS mRNA showed a different pattern (table 3). ADAMTS-5 mRNA levels were lower in both dysfunctional PTT and FDLT than in normal PTT (figure 2E). ADAMTS-1 mRNA levels in dysfunctional PTT were lower than in FDLT, although neither of these groups showed a significant difference from normal PTT (figure 2F). ADAMTS-4 mRNA showed no substantial difference between the three tendon groups, although a small increase $(20 \%)$ in median values in dysfunctional PTT compared with the paired FDLT samples was statistically significant (table 3).

\section{Analysis of PTT and FDLT from male patients}

The male samples also showed significant biochemical and mRNA differences between clinical groups: (A) fourfold higher levels of GAG in dysfunctional PTT than in FDLT $(p<0.05)$; (B) more than twofold higher levels of pentosidine in FDLT than in dysfunctional PTT $(\mathrm{p}=0.01)$; (C) lower levels of OHPyr cross-links in FDLT than in either normal or dysfunctional PTT ( $p=0.05$ and $p=0.01$ respectively); (D) higher levels of HHL and HLNL cross-links in FDLT than in either normal or dysfunctional PTT ( $\mathrm{p} \leq 0.01$ in each comparison); (E) higher expression of mRNA encoding types I and III collagen, 
(A) type I collagen

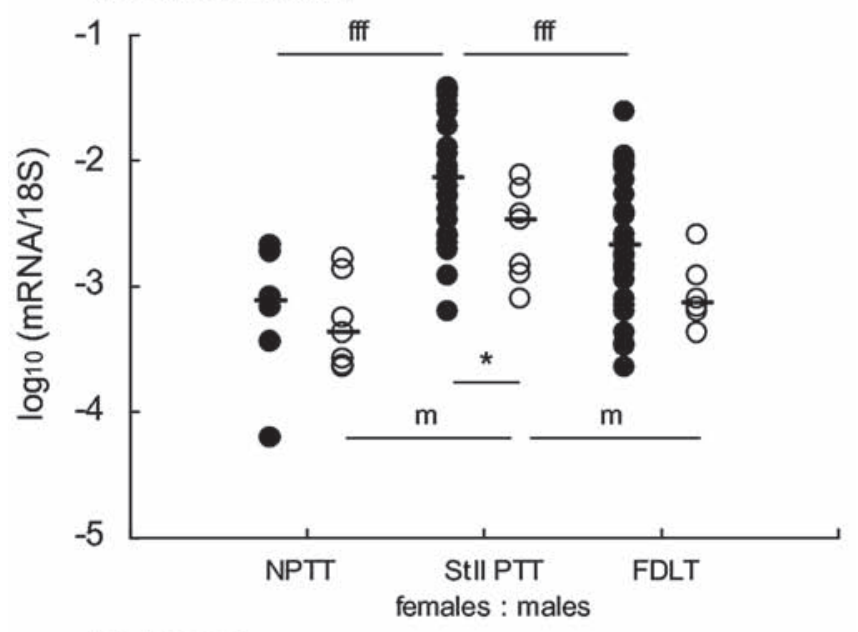

(B) MMP-2

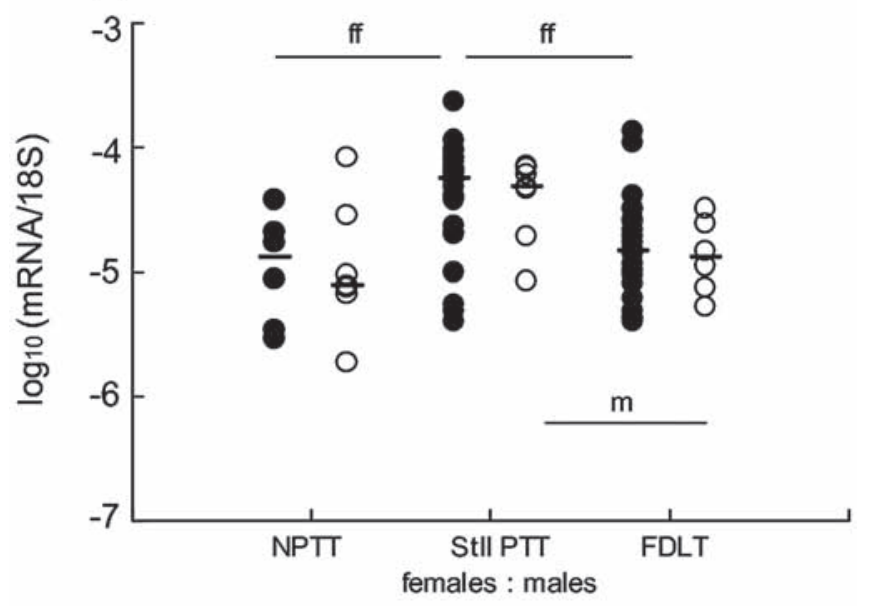

(C) MMP-3

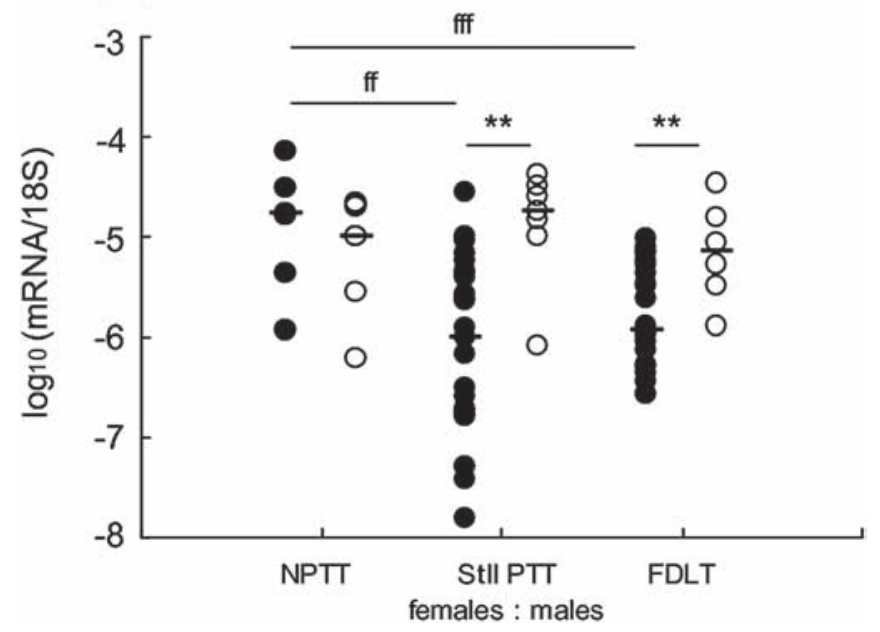

Figure 3 Comparison of mRNA expression in female (closed circle) and male (open circle) posterior tibialis tendon (PTT) and flexor digitorum longus tendon (FDLT). Tendon RNA samples were analysed for specific mRNA, expressed relative to $18 \mathrm{~S}$ rRNA as outlined in the text. Each point represents an individual sample. The median values are shown (horizontal bar). Significant differences between clinical groups for female patients are indicated ${ }^{\mathrm{fff}} \mathrm{p}<0.001$, ${ }^{\mathrm{ff}} \mathrm{p}<0.01$, and for male patients are indicated $\mathrm{m}_{\mathrm{p}}<0.05$. Significant differences between females and males of the same clinical group are indicated ${ }^{* *} p<0.01,{ }^{*} p<0.05$. MMP, matrix metalloproteinase; N, normal; St II, stage II. aggrecan, biglycan, versican and MMP-2 mRNA in dysfunctional PTT compared with normal PTT and/or FDLT (figure $3 \mathrm{~A}, \mathrm{~B}$ and data not shown).

Each of these differences in the male sample set was similar to that observed in female patients, and there were no significant differences between men and women in total collagen, total GAG, collagen modifications and most mRNA, in any of the three clinical groups (data not shown and figure 3). Indeed, combining the male and female datasets for many parameters increased the statistical significance of the differences observed between clinical groups, compared with the female data alone, irrespective of whether the male data analysed separately showed significant changes. However, certain mRNA differences were observed between men and women. Levels of type I collagen mRNA in dysfunctional PTT and type III collagen mRNA in FDLT were lower in men than in women, although this did not affect the pattern of higher expression in dysfunctional PTT than in the other clinical groups (figure $3 \mathrm{~A}$ ). In contrast, MMP-3 mRNA was expressed at higher levels in both dysfunctional PTT and FDLT in men compared with women (figure 3C), and there were no differences between the clinical groups for men (figure $3 \mathrm{C}$ ), in contrast with the marked differences observed in women (figure 3 ).

\section{DISCUSSION}

Differences in matrix composition and cross-link content are found between functionally distinct tendons, and at different sites within tendons. Many of these changes reflect different patterns of loading and matrix turnover, although the relationship to the tendon material properties is uncertain. ${ }^{10} 1113$ The PTT is a load-bearing tendon, and the normal specimens in this study were obtained from the region that wrapped around the medial malleolus, and were therefore exposed to some compression, at least on the inner surface. ${ }^{21}$ The FDLT is predominantly a 'positional' tendon, normally not heavily loaded, and the specimens used in this study were obtained from a slip close to the bone insertion which received predominantly tensile loading. Although the normal PTT and FDLT specimens were taken from patients with lower limb conditions, so that effects of reduced mobility on their phenotype cannot be excluded (see Methods), both groups showed normal morphological and histological appearance (table 1). Furthermore, the increase in pentosidine content with age in normal PTT and FDLT (figure 1) is similar to that previously reported in biceps brachii and patellar tendons, ${ }^{10-12}$ indicating that each of these tendons has a stable and long-lived matrix structure in which advanced glycation end products on collagen (and other longlived proteins) can accumulate linearly with time.

Although many parameters in the normal PTT and FDLT were similar (tables 2 and 3), there were some marked differences between them. The FDLT contained lower levels of the ketoimine-derived mature cross-link OHPyr than normal PTT and higher levels of the aldimine-derived mature cross-links HHL and HLNL (table 2 and figure 1). Aldimine-derived cross-links are generally more abundant in tendons, whereas ketoiminederived cross-links are typically higher in bone and cartilage. ${ }^{26}$ OHPyr is more abundant in weight-bearing and highly stressed tendons, ${ }^{13}$ such as the supraspinatus tendon in the rotator cuff, which is subjected to compressive loads in addition to tension. ${ }^{10}$ The cross-link profile of FDLT is broadly similar to other positional tendons, whereas the higher OHPyr content of normal PTT suggests (by analogy with other tendons) ${ }^{10} 13$ adaptation to compression against the medial malleolus. The FDLT also contained lower levels of GAG and of ADAMTS-5 mRNA than 
normal PTT (tables 1 and 2), indicating a different proteoglycan composition in the FDLT, possibly requiring lower maintenance turnover than the normal PTT. The fact that the FDLT is used successfully to replace dysfunctional PTT may indicate either that the tendon rapidly adapts to its new location, changing the synthesis of matrix components, or these differences in composition do not significantly affect the material properties of the tendon.

Both the biochemical and mRNA expression changes described here for stage II dysfunctional PTT can be compared with previous work in other tendons. The lower than expected pentosidine levels in specimens from dysfunctional PTT (figure 1) are similar to those observed in degenerate supraspinatus tendons ${ }^{10} 18$ and Achilles tendinopathy. ${ }^{27}$ The reduction in pentosidine/collagen may result from reversal of the modification, removal of old (modified) collagen, or insertion of new (unmodified) collagen. In supraspinatus tendons, replacement of the original collagen matrix was additionally supported by measurement of the racemisation of aspartate, a marker of protein residence time. ${ }^{10} 18$ In dysfunctional PTT, synthesis of new collagen matrix is indicated by the observed increase in levels of mRNA encoding both types I and III collagens (table 3 ) and by increased levels of both types III and V collagen proteins. ${ }^{23}$ Furthermore, increased removal of old collagen is likely, given the increased expression of mRNA encoding the matrix-degrading enzymes MMP-2 (72 $\mathrm{kDa}$ gelatinase) and MMP-13 (collagenase 3), which were also elevated in Achilles tendinopathy. ${ }^{19}$ Both these enzymes have activity against a variety of potential substrates, including the main structural component of tendon, fibrillar type I collagen. Taken together, our data indicate that there is a chronic remodelling of the original collagen matrix.

The higher GAG content in dysfunctional PTT is a common observation in chronic tendinopathy, ${ }^{16} 2328$ and has been associated with increased deposition of proteoglycans including aggrecan and biglycan. ${ }^{29}$ Indeed, the median ratio of aggrecan mRNA/versican mRNA increased from 0.37 in normal PTT to 1.83 in dysfunctional PTT, which is very similar to the increase observed in chronic Achilles tendinopathy. ${ }^{17}$ These changes in proteoglycan expression may indicate a process of fibrocartilaginous change induced by forces of shear and/or compression acting on the tenocytes, and a switch in cell phenotype from tenocyte to chondrocyte. However, expression of type II collagen mRNA in dysfunctional PTT remained 100- to 1000-fold lower than that of type I and type III (data not shown), which suggests only a partial phenotypic change. Alternatively, the increased production of aggrecan and biglycan may be secondary to injury or part of the chronic remodelling response, potentially stimulated by the activity of cytokines and growth factors such as transforming growth factor $\beta .^{30}$

In addition to increased aggrecan and biglycan expression, the increased proteoglycan content observed in dysfunctional PTT may result in part from a decrease in ADAMTS-5 mRNA expression (table 2). The proteolytic processing of proteoglycans in tendons is evidently a normal process in healthy tendon, ${ }^{31} 32$ and recent evidence from ADAMTS-5-/- mice indicates that ADAMTS-5 aggrecanase activity is required for the maintenance of normal tendon structure and some biomechanical properties. ${ }^{33}$ Both the FDLT and Achilles tendons in the ADAMTS-5-/- mice showed increased aggrecan deposition, but the biomechanical changes observed differed between the two tendons. ${ }^{33}$

The functional significance of decreased expression of MMP-3 mRNA and increased expression of MMP-23 and ADAM-12 mRNA in dysfunctional PTT is hard to interpret, since the main substrates of these enzymes in tendon are as yet unknown. The expression of MMP-3 mRNA was one of the few parameters to show differences between men and women: lower in dysfunctional PTT (and FDLT) in women but not in men (figure $3 \mathrm{C}$ ). Whether this gender difference in MMP-3 expression is relevant to the higher incidence of PTT dysfunction in women requires further investigation; previous studies have shown a reduction in MMP-3 expression in Achilles tendinopathy (in men). ${ }^{19}{ }^{34}$ Studies on the potential function(s) of MMP-23 have shown some correlation with endochondral ossification and the ability to bind and inhibit potassium channels. ${ }^{3536} \mathrm{ADAM}-12$ is linked to tumorigenesis, myogenesis and adipogenesis, and appears to have multiple roles in the processing of growth factors and their receptors. ${ }^{37}$ Thus both enzymes may be associated with the changes in cell phenotype found in tendinopathy, although ADAM-12 expression is, unusually, increased in ruptured tendons as well as in tendinopathy. ${ }^{19} 38$ As with other metalloproteinases, consideration must also be given to the extensive post-translational regulation of these enzymes by highly controlled processes of pro-enzyme activation and subsequent inhibition by Tissue Inhibitor of Metalloproteinases and other inhibitors in the tissue. ${ }^{39} 40$ Further studies are required to determine whether these enzymes are proteolytically active in chronic tendinopathy.

Stage II PTT dysfunction and painful Achilles tendinopathy have different clinical presentations: although both involve swelling and pain, PTT dysfunction presents with deformity and flattening of the foot, whereas deformity is not a clinical problem in Achilles tendinopathy. It is therefore interesting that the gene expression pattern in stage II PTT is in many respects similar to that observed in Achilles tendinopathy. ${ }^{17} 19$ In contrast, ruptured Achilles tendons showed substantially different changes in enzyme and matrix gene expression, ${ }^{17} 19$ which may be the consequence and not the cause of the rupture. The molecular differences are consistent with the histological observations (see table 1) that showed similar features in stage II PTT to those observed in painful Achilles tendinopathy, and very different from the typical histology of ruptured tendons. ${ }^{16} 1941$ Increased expression of MMP-2, MMP-13, collagen and aggrecan and decreased MMP- 3 have also been reported in torn rotator cuff tendons. ${ }^{42-45}$ However, these are often fullthickness tears that also show decreased expression of decorin and increased MMP-9, ${ }^{43-45}$ more similar to ruptured Achilles tendons. ${ }^{17} 19$ Taken together, the data suggest that the underlying processes in the chronic Achilles and PTT conditions are the same; the presentational differences may simply reflect the different functions of the two musculotendinous structures: maintenance of the foot arch versus push-off during gait. A difference in biomechanical stability, rather than tendon structure, may also underlie the greater susceptibility of women to PTT dysfunction, since many of the biomolecular parameters appear similar in men and women.

In summary, the 'stretching out' of the PTT is accompanied by a process of chronic matrix remodelling, which may be indicative of an incomplete healing response to repeated micro-injury or 'overuse'. Treatment strategies designed to resolve or enhance the chronic remodelling response are likely to prove beneficial in both Achilles and posterior tibialis tendinopathy.

Acknowledgements We thank Dr Helen Birch (Royal National Orthopaedic Hospital Stanmore, University College London) for the provision of specimens of normal tendon tissues, and Dr Kirsten Legerlotz for useful comments on the manuscript.

Funding This work was supported by Arthritis Research UK (17826), Cambridge Arthritis Research Endeavour (CARE), The Rosetrees Trust, The HB Allen Trust and the Sybil Eastwood Trust. 
Competing interests None.

Patient consent Obtained.

Ethics approval Cambridge.

Provenance and peer review Not commissioned; externally peer reviewed.

\section{REFERENCES}

1. Rees JD, Wilson AM, Wolman RL. Current concepts in the management of tendon disorders. Rheumatology (Oxford) 2006;45:508-21.

2. Kohls-Gatzoulis J, Angel JC, Singh D, et al. Tibialis posterior dysfunction: a common and treatable cause of adult acquired flatfoot. BMJ 2004;329:1328-33.

3. Johnson KA, Strom DE. Tibialis posterior tendon dysfunction. Clin Orthop Relat Res 1989;239:196-206.

4. Funk DA, Cass JR, Johnson KA. Acquired adult flat foot secondary to posterior tibialtendon pathology. J Bone Joint Surg Am 1986;68:95-102.

5. Gluck GS, Heckman DS, Parekh SG. Tendon disorders of the foot and ankle, part 3: the posterior tibial tendon. Am J Sports Med 2010;38:2133-44.

6. Wacker JT, Hennessy MS, Saxby TS. Calcaneal osteotomy and transfer of the tendon of flexor digitorum longus for stage-II dysfunction of tibialis posterior. Three- to five-year results. J Bone Joint Surg Br 2002;84:54-8.

7. Lee $\mathbf{D}$. Effects of posterior tibial tendon augmented with biografts and calcaneal osteotomy in stage II adult-acquired flatfoot deformity. Foot Ankle Spec 2009;2:27-31.

8. Riley G. The pathogenesis of tendinopathy. A molecular perspective. Rheumatology (Oxford) 2004; 43:131-42.

9. Riley G. Tendinopathy-from basic science to treatment. Nat Clin Pract Rheumatol 2008;4:82-9.

10. Bank RA, TeKoppele JM, Oostingh G, et al. Lysylhydroxylation and non-reducible crosslinking of human supraspinatus tendon collagen: changes with age and in chronic rotator cuff tendinitis. Ann Rheum Dis 1999;58:35-41.

11. Hansen $\mathbf{P}$, Haraldsson BT, Aagaard P, et al. Lower strength of the human posterior patellar tendon seems unrelated to mature collagen cross-linking and fibril morphology. J Appl Physiol 2010;108:47-52.

12. Couppé $\mathbf{C}$, Hansen $\mathrm{P}$, Kongsgaard M, et al. Mechanical properties and collagen crosslinking of the patellar tendon in old and young men. J App/ Physiol 2009;107:880-6.

13. Thorpe CT, Streeter I, Pinchbeck GL, et al. Aspartic acid racemization and collagen degradation markers reveal an accumulation of damage in tendon collagen that is enhanced with aging. J Biol Chem 2010;285:15674-81.

14. Waggett AD, Ralphs JR, Kwan AP, et al. Characterization of collagens and proteoglycans at the insertion of the human Achilles tendon. Matrix Biol 1998;16:457-70.

15. Benjamin M, Kumai T, Milz S, et al. The skeletal attachment of tendons-tendon "entheses". Comp Biochem Physiol, Part A Mol Integr Physiol 2002;133:931-45.

16. Movin T, Gad A, Reinholt FP, et al. Tendon pathology in long-standing achillodynia Biopsy findings in 40 patients. Acta Orthop Scand 1997;68:170-5.

17. Corps AN, Robinson AH, Movin T, et al. Increased expression of aggrecan and biglycan mRNA in Achilles tendinopathy. Rheumatology (Oxford) 2006;45:291-4.

18. Riley GP, Curry V, DeGroot J, et al. Matrix metalloproteinase activities and their relationship with collagen remodelling in tendon pathology. Matrix Biol 2002;21:185-95.

19. Jones GC, Corps AN, Pennington CJ, et al. Expression profiling of metalloproteinases and tissue inhibitors of metalloproteinases in normal and degenerate human achilles tendon. Arthritis Rheum 2006;54:832-42.

20. Moriggl B, Kumai T, Milz S, et al. The structure and histopathology of the "enthesis organ" at the navicular insertion of the tendon of tibialis posterior. J Rheumatol 2003; 30:508-17.

21. Petersen W, Hohmann G, Pufe T, et al. Structure of the human tibialis posterior tendon. Arch Orthop Trauma Surg 2004;124:237-42.
22. Mosier SM, Lucas DR, Pomeroy G, et al. Pathology of the posterior tibial tendon in posterior tibial tendon insufficiency. Foot Ankle Int 1998;19:520-4.

23. Gonçalves-Neto J, Witzel SS, Teodoro WR, et al. Changes in collagen matrix composition in human posterior tibial tendon dysfunction. Joint Bone Spine 2002;69:189-94.

24. Fowble VA, Vigorita VJ, Bryk E, et al. Neovascularity in chronic posterior tibial tendon insufficiency. Clin Orthop Relat Res 2006;450:225-30.

25. Riley GP, Goddard MJ, Hazleman BL. Histopathological assessment and pathological significance of matrix degeneration in supraspinatus tendons. Rheumatology (Oxford) 2001;:40:229-30.

26. Robins SP. Biochemistry and functional significance of collagen cross-linking. Biochem Soc Trans 2007;35(Pt 5):849-52.

27. de Mos M, van El B, DeGroot J, et al. Achilles tendinosis: changes in biochemical composition and collagen turnover rate. Am J Sports Med 2007;35:1549-56.

28. Riley GP, Harrall RL, Constant CR, et al. Glycosaminoglycans of human rotator cuff tendons: changes with age and in chronic rotator cuff tendinitis. Ann Rheum Dis 1994;53:367-76.

29. Samiric T, Parkinson J, llic MZ, et al. Changes in the composition of the extracellular matrix in patellar tendinopathy. Matrix Biol 2009;28:230-6.

30. Vogel KG, Hernandez DJ. The effects of transforming growth factor-beta and serum on proteoglycan synthesis by tendon fibrocartilage. Eur J Cell Biol 1992;59:304-13.

31. Rees SG, Flannery CR, Little CB, et al. Catabolism of aggrecan, decorin and biglycan in tendon. Biochem J 2000;350 Pt 1:181-8.

32. Samiric T, Ilic MZ, Handley CJ. Characterisation of proteoglycans and their catabolic products in tendon and explant cultures of tendon. Matrix Biol 2004;23:127-40.

33. Wang VM, Bell RM, Thakore $\mathrm{R}$, et al. Murine tendon function is adversely affected by aggrecan accumulation due to the knockout of ADAMTS5. J Orthop Res. Published Online First: 16 September 2011. doi:10.1002/jor.21558.

34. Ireland D, Harrall R, Curry V, et al. Multiple changes in gene expression in chronic human Achilles tendinopathy. Matrix Biol 2001;20:159-69.

35. Clancy BM, Johnson JD, Lambert AJ, et al. A gene expression profile for endochondral bone formation: oligonucleotide microarrays establish novel connections between known genes and BMP-2-induced bone formation in mouse quadriceps. Bone 2003;33:46-63.

36. Rangaraju S, Khoo KK, Feng ZP, et al. Potassium channel modulation by a toxin domain in matrix metalloprotease 23. J Biol Chem 2010;285:9124-36.

37. Kveiborg M, Albrechtsen R, Couchman JR, et al. Cellular roles of ADAM12 in health and disease. Int J Biochem Cell Biol 2008;40:1685-702.

38. Jelinsky SA, Rodeo SA, Li J, et al. Regulation of gene expression in human tendinopathy. BMC Musculoskelet Disord 2011:12:86.

39. Ra HJ, Parks WC. Control of matrix metalloproteinase catalytic activity. Matrix Biol 2007;26:587-96.

40. Murphy G, Willenbrock F, Crabbe T, et al. Regulation of matrix metalloproteinase activity. Ann N Y Acad Sci 1994;732:31-41.

41. Kannus $\mathbf{P}$, Józsa L. Histopathological changes preceding spontaneous rupture of a tendon. A controlled study of 891 patients. J Bone Joint Surg Am 1991;73:1507-25.

42. Lo IK, Marchuk LL, Hollinshead R, et al. Matrix metalloproteinase and tissue inhibitor of matrix metalloproteinase mRNA levels are specifically altered in torn rotator cuff tendons. Am J Sports Med 2004;32:1223-9.

43. Lo IK, Boorman R, Marchuk L, et al. Matrix molecule mRNA levels in the bursa and rotator cuff of patients with full-thickness rotator cuff tears. Arthroscopy 2005;21:645-51.

44. Lakemeier $\mathbf{S}$, Braun J, Efe T, et al. Expression of matrix metalloproteinases 1, 3, and 9 in differing extents of tendon retraction in the torn rotator cuff. Knee Surg Sports Traumatol Arthrosc 2011;19:1760-5

45. Shindle MK, Chen CC, Robertson C, et al. Full-thickness supraspinatus tears are associated with more synovial inflammation and tissue degeneration than partialthickness tears. J Shoulder Elbow Surg 2011;20:917-27. 